\title{
Oportunidades y trampas de las redes globales de producción: un análisis del sector petrolero en Bolivia
}

Prospects and pitfalls of global production networks: an analysis of Bolivia's oil and gas sector

\author{
Historial del artículo \\ Recibido: \\ 10 de enero de 2021 \\ Revisado
}

24 de mayo de 2021

Aceptado:

01 de junio de 2021

\author{
Sören Scholvin ${ }^{\mathrm{a}}$ \\ a Instituto de Geografía Económica y Cultural, Universidad de Hanover, Alemania. Correo electrónico: scholvin@wigeo. \\ uni-hannover.de \\ ORCID: https://orcid.org/0000-0001-5911-2718
}

\section{Palabras clave}

Desarticulación, red global de producción, Santa Cruz, sector petrolero.

\section{Keywords}

Disarticulation, global production network, oil and gas sector, Santa Cruz.

\section{Resumen}

Este artículo analiza las perspectivas del desarrollo regional a través del sector petrolero en Bolivia. Se aplica el enfoque de las redes globales de producción y se estudian tres procesos de "desarticulaciones". Mientras que algunas empresas en la ciudad de Santa Cruz se benefician de la integración en el sector petrolero, la economía regional se ve también afectada por la degradación de estas empresas, su expulsión del sector y la no participación de otras. En base a dieciséis entrevistas con actores clave e información de fuentes secundarías, el autor explica que este desarrollo negativo resulta de la transformación de las redes correspondientes hacia un modelo "llave en mano" que aventaja a epecistas extranjeras, también denominadas "empresas EPCM". No obstante, concluye que hay que reconocer que la participación en la economía global genera tanto inconvenientes como oportunidades -un hecho que indica que falta una teoría que explique bajo qué condiciones aplican los procesos de desarticulaciones-.

\begin{abstract}
This article analyses the prospects of regional development through the oil and gas sector in Bolivia. The author applies the global production networks approach and investigates three processes of 'disarticulations'. While some firms in the city of Santa Cruz benefit from their integration into the oil and gas sector, the regional economy is also affected by the downgrading of these firms, their exclusion from the sector, and the non-participation of others. Based on 16 interviews with key players and information from secondary sources, the author explains that this negative development results from the transformation of the corresponding networks towards a 'turn-key' model, which is beneficial to foreign engineering, procurement, construction, and management (EPCM) companies. He concludes, however, that one must recognise that participation in the global economy creates inconveniences and opportunities - a fact that indicates that there is need for a theory that explains under which conditions the processes of disarticulacions apply-.
\end{abstract}




\section{Introducción}

En junio 2019, los gobiernos de Bolivia y Perú anunciaron un "megapuerto" que será construido en la ciudad de Ilo en el sur de Perú. Más allá del tema del acceso al océano, la cooperación de ambos países se basa en las exportaciones de gas natural. Bolivia ya exporta gas natural a los países limítrofes, pero el proyecto de Ilo promete llegar a un puerto donde se puede embarcar gas natural licuado para los mercados asiáticos, principalmente la India, cuyo gobierno ha acordado invertir USD 10.000 millones en una planta de licuefacción en Bolivia y el gasoducto hacia Ilo. En otras palabras, los hidrocarburos son esenciales para la economía y política boliviana. Como demuestra el presente artículo, la relevancia económica no se limita a las exportaciones. Aborda también la transición de la mera extracción de recursos naturales a actividades más lucrativas, especialmente la industria de suministros. Con este trasfondo, el artículo responde a la pregunta siguiente: ¿cuáles son las perspectivas del desarrollo regional a través del sector petrolero en Bolivia?

Se aplica el enfoque de las redes globales de producción, es decir, un enfoque que se ha transformado en un pilar para los estudios de la geografía económica durante las dos últimas décadas. Con base en una heurística de redes, este enfoque enfatiza la diversidad de las conexiones horizontales y verticales entre los actores que son relevantes para el proceso económico en cuestión (Coe et al., 2008; en cuanto a la heurística de redes, véase también: Dicken et al., 2001). Es altamente inclusivo con respecto a todo lo que da forma a la economía global y, por lo tanto, influye sobre las trayectorias de desarrollo que marcan las regiones. En un solo estudio no se puede aplicar este enfoque en su totalidad. Presta varias herramientas analíticas y cada científico debe escoger algunas -las que le parezcan más adecuadas - para el asunto empírico bajo consideración. El presente artículo se concentra en la captura de valor, el poder de diferentes tipos de empresas y el "acoplamiento estratégico" (Coe \& Yeung, 2015; Yeung, 2009).

Como se explica a continuación, el enfoque de las redes globales de producción tiende a limitarse al desarrollo exitoso. Escasamente se toman en consideración los fracasos. Sin embargo, las industrias extractivas raramente facilitan el desarrollo regional, como demuestran, entre otros autores, Atienza et al. (2016, 2021), Barrat \& Ellem (2019) y Mackinnon (2013) en sus artículos sobre la minería en Antofagasta (Chile) y el Pilbara (Australia), respectivamente. Por lo tanto, este artículo responde al llamado de Phelps et al. (2018) de involucrarse con "el lado oscuro de la geografía económica". Se aplica el concepto de "desarticulaciones" - desarrollado por Bair \& Werner (2011), McGrath (2018) y Werner (2016)para mostrar cómo el sector petrolero en Bolivia se ha visto afectado por procesos de degradación, expulsión y no participación de/en las redes globales. Significa que más allá de los hallazgos empíricos, el artículo avanza también sobre el debate teórico sobre las redes globales de producción, extendiendo el enfoque tradicional hacia una perspectiva crítica.

Como el artículo enfoca la industria de suministros, los lectores deben tener en cuenta que varios temas ciertamente importantes no se abordan. Esto incluye, en particular, el debate sobre la maldición de los recursos (véase: Hinojosa et al., 2015; Ramírez Cendero, 2014), el impacto de la extracción de hidrocarburos sobre las comunidades indígenas y el medio ambiente (véase: Hope, 2016; Pellegrini \& Ribera Arismendi, 2012), así como las luchas políticas relacionadas con los recursos naturales (véase: Andreucci, 2017; Carrizo \& Ramousse, 2010).

El artículo consiste en tres secciones principales. Primero, se introduce el enfoque de las redes globales de producción, elaborando especialmente sobre las desarticulaciones. Segundo, se resume la metodología. La tercera sección presenta los hallazgos empíricos. El artículo concluye con una breve sugerencia en cuanto al concepto de desarticulaciones.

\section{Marco conceptual}

El análisis de cualquier red de producción empieza con la creación y el aumento de valor (Henderson et al., 2002) -por ejemplo, recursos naturales que son extraídos y procesados- ${ }^{-}$. No obstante, una cosa es crear y aumentar valor, pero otra es capturar este valor. Como observan Kaplinsky (2005) y Milberg \& Winkler (2013), el Sur global se caracteriza por poca captura de valor. Coe $\&$ Yeung proponen que la capacidad correspondiente resulta del poder de las empresas que interactúan en las redes globales. Estas redes "pueden entenderse como una serie de relaciones de intercambio. Las variaciones en el equilibrio de poder a lo largo de la[s] red[es] afectan la capacidad de cada empresa en capturar valor" (2015, p. 17). ¿Por qué es tan importante la captura de valor? Coe \& Yeung argumentan que "para el objetivo del desarrollo [regional], el valor debe retenerse adentro de las empresas, o [por lo menos] en las partes de las empresas, con sede en la región en cuestión" (2015, p. 172).

Es obvio que no todas las compañías tienen el mismo poder y, 
en consecuencia, hay diferencias considerables con respecto al valor que capta cada una. Según Coe \& Yeung (2015), el poder y, por ende, la captura de valor, dependen de las tareas realizadas por cada empresa. Distinguen entre las empresas líderes, sus socios estratégicos, los proveedores especializados y los proveedores genéricos. Mientras que las empresas líderes controlan las redes como, por ejemplo, las operadoras en el sector petrolero (Petrobras, Repsol, Total y otras), los socios estratégicos ofrecen soluciones completas o al menos parciales mediante el codiseño o desarrollo de nuevos productos y servicios. Los proveedores especializados proporcionan bienes intermedios. Por esa razón, ejercen menos poder y capturan menos valor que los socios estratégicos. Los proveedores genéricos suministran productos y servicios altamente estandarizados y de bajo valor que escasamente tienen una dedicación específica a la red o al sector bajo consideración. Por lo tanto, los proveedores genéricos son aún menos poderosos y capturan aún menos valor.

Como las economías regionales muchas veces se caracterizan por la predominancia de un tipo de empresa según el guion que se acaba de presentar, se pueden distinguir tres formas del acoplamiento estratégico, es decir, tres maneras de cómo las regiones se conectan con la economía global (Coe \& Yeung, 2015; Yeung, 2009):

- $\quad$ Empresas locales se internacionalizan para participar en las redes de producción (o crear nuevas). Se tornan líderes o, por lo menos, sirven como socios estratégicos, para prestar productos y servicios innovadores. Como se ha señalado, ambos roles implican una captura de valor relativamente alta. En cuanto al desarrollo regional, esta forma de acoplamiento estratégico es la más prometedora, como lo han demostrado, por ejemplo, la industria eólica en Alemania, Noruega y el Reino Unido (Mackinnon et al., 2019), así como los sectores automotrices y electrónicos en Asia Oriental (Yeung, 2016).

- $\quad$ Empresas locales que son especializadas para ciertas tareas y cumplen con los requerimientos de líderes no locales están integradas en algunas redes. Aunque sus productos y servicios solo sean insumos en procesos de producción fuera de sus propias capacidades, hay posibilidades de pasar a la primera forma del acoplamiento estratégico (Van Grunsven \& Hutchinson, 2016; Yeung, 2016). Al mismo tiempo, existe una clara jerarquía entre las empresas líderes (que controlan las redes correspondientes) y las empresas locales (que asumen un rol subordinado). Esta jerarquía tiene implicaciones para la captura de valor.

- Corporaciones no locales integran la región bajo consideración en las redes que controlan, contratando a proveedores locales que prestan mano de obra y productos y servicios genéricos. La captura de valor al nivel regional disminuye mucho. Algunos científicos proponen que esta forma del acoplamiento estratégico puede servir como base del desarrollo regional (Kaplinsky \& Morris, 2016; Morris et al., 2012), mientras que otros apuntan al estancamiento en la provisión de productos y servicios genéricos (Scholvin, 2019; Scholvin et al., 2020).

Aunque los párrafos anteriores hayan presentado conceptos y términos que sirven para explicar tanto el desarrollo exitoso de algunas regiones, como el fracaso de otras, al leer las publicaciones seminales sobre las redes globales de producción, apenas se aprende que las regiones pueden estancarse o tener un rendimiento peor porque participan en la economía global. Coe \& Yeung (2015) mencionan resultados negativos como la diferenciación intrarregional y el permanecer al "acoplamiento estructural", es decir, la tercera de las formas recién mencionadas. Como observan Phelps et al., "las palabras [']desarrollo desigual ['] ocurren con frecuencia en [el libro de Coe \& Yeung], pero son en gran parte palabras de una posibilidad teórica implícita en vez de [una posibilidad] explícita o una realidad empírica" (2018, p. 240).

Como se concentra en el desarrollo exitoso, la investigación sobre las redes globales de producción sufre de lo que Bair \& Werner (2011) llaman "el sesgo inclusivo". Enfoca a las empresas líderes y sus redes, como se demuestra probablemente mejor por el artículo de Henderson et al. (2002) y el libro de Coe \& Yeung (2015) -ambos han guiado los debates académicos, sirviendo como orientación en prácticamente cada estudio-. Los proveedores subordinados y, aún más importante, las empresas, personas y regiones que no logran vincularse a las redes globales son de relevancia secundaria. Muchas veces quedan completamente fuera del panorama analítico. Raramente se investiga cómo y por qué algunos quedan atrás o no se benefician de las dinámicas de la economía global.

Para superar al sesgo inclusivo, Bair \& Werner (2011), McGrath (2018) y Werner (2016) plantean una perspectiva de desarticulaciones (que, en parte, va más allá de lo que se estudia en el presente artículo). Aplicando esta idea, Murphy explica que "las desarticulaciones son visibles en las exclusiones, los procesos de degradación [y] empobrecimiento [...] que pueden ocurrir juntos con los acoplamientos a las redes globales" (2019, p. 950). Como lo indica su análisis del sector del turismo en Zanzíbar (Tanzania), las desarticulaciones comprenden 
tres procesos:

- Algunas empresas y personas nunca se conectan a las redes globales, compartiendo el mismo espacio físico con compañías globalizadas y prósperas, pero sin oportunidades de aprovechar de alguna forma de colaboración. Los complejos hoteleros de lujo de propiedad extranjera en Zanzíbar reciben a turistas acaudalados y generan enormes ganancias, mientras que el 30 por ciento de la población local vive por debajo del umbral de pobreza y el 15 por ciento de los hogares sufre de inseguridad alimentaria severa.

- Compañías e individuos pueden ser expulsados de las redes globales de producción. Los pequeños hoteles de propriedad local en Zanzíbar, que hasta hace un tiempo fueron frecuentados por viajeros aventureros, son percibidos como poco confiables por los turistas que ahora visitan la isla. No pueden pagar las comisiones cobradas por sitios web como TripAdvisor. Los guías locales dependen de los clientes sin cita previa, pero se ven ignorados por los operadores del extranjero que ofrecen paquetes turísticos completos.

- Hay también empresas que permanecen adentro de las redes, pero sufren de una degradación (véase también: Gibbon \& Ponte, 2005). En Zanzíbar, las empresas locales han pasado a emplear conductores, guías y otro personal según sea necesario. Como explica Kaplinsky (2000), este proceso es debido al hecho de que el valor resulte cada vez más de los activos intangibles, como la propiedad intelectual, el acceso a la financiación y la capacidad de coordinar las redes globales, siendo fortalezas típicas de las empresas del Norte global.

Viendo estas trampas de las redes globales de producción, Bair, McGrath, Werner y probablemente también Murphy entienden la degradación, la expulsión y la no participación de algunos actores y regiones como una condición sine qua non para la inclusión y el suceso de otros. En otras palabras, las redes globales de producción reflejan un cambio constante entre qué y quién está incorporado o excluido (Bair et al., 2013). McGrath enfatiza en este sentido que "la perspectiva de des/articulaciones [...] cuestiona las trayectorias [eufemísticas] del desarrollo capitalista" (2019, p. 9). Por lo tanto, la investigación que tiene como objetivo superar el sesgo inclusivo trata de cómo "hacer que las localizaciones y personas sean exteriores a las redes globales de producción forma parte integral de la acumulación continua a través de estos arreglos y la reproducción del desarrollo desigual que conllevan" (Werner, 2016, p. 458). Después del resumo de la metodología, aplico esta idea al sector petrolero en Bolivia.

\section{Materiales y métodos}

El análisis se basa en dieciséis entrevistas narrativas con representantes de autoridades públicas, organizaciones empresariales y, lo más importante, empresas bolivianas y extranjeras. Considerando el enfoque del artículo en el acoplamiento estratégico y su lado oscuro, la selección de entrevistados refleja la diversidad de los actores clave. Es decir que la metodología cumple con el criterio de representatividad en el marco de lo que es posible para una investigación cualitativa. Las entrevistas se realizaron en Santa Cruz. La identificación de los entrevistados fue a través de sus páginas personales en LinkedIn y por el sistema de la bola de nieve. La Cámara de Hidrocarburos de Bolivia ayudó a generar el contacto con algunos entrevistados.

Hubo tres guías de preguntas - para compañías transnacionales, proveedores santacruceños y asociaciones empresariales/autoridades públicas (véase abajo)- que fueron modificadas ligeramente antes de cada entrevista, reflejando la especialización del entrevistado y la naturaleza exacta de su empresa u organización. El objetivo de las entrevistas era entender mejor tanto la organización territorial del sector petrolero, enfocando el rol de Santa Cruz, como las relaciones entre diferentes empresas, especialmente con respecto a los proveedores locales. En total, trece entrevistas fueron grabadas. Luego, fueron estructuradas a lo largo de oportunidades para el desarrollo regional y los tres procesos de desarticulaciones mencionados en la sección anterior. En tres casos, solo se pudo tomar notas. La mayoría de los entrevistados no hablaron como representantes oficiales de su empresa u organización, pero más abajo se brinda la afiliación correspondiente para contextualizar la información prestada por los entrevistados.

\section{Guías de preguntas}

Guía: empresas transnacionales

1. Me interesa cómo su empresa organiza el trabajo. ¿Qué actividades se realizan acá en Santa Cruz?

2. ¿Cómo se toman las decisiones en su empresa, especialmente en términos de las relaciones con la casa matriz?

3. ¿Qué ventajas de ubicación le ofrece Santa Cruz?

4. Me gustaría saber más sobre sus relaciones con otras compañías. ¿Podría darme una descripción general de 
sus proveedores? ¿Qué tipo de productos y servicios prestan ellos? ¿Cómo se benefician de la interacción con su empresa?

5. ¿Qué actividades y proyectos quieren realizar en Santa Cruz en los próximos años? ¿Cuáles están previstos para otros lugares en Bolivia?

Guía: proveedores locales

1. Su empresa presta servicios al sector petrolero. ¿En qué países/regiones está activa?

2. ¿Qué ventajas de ubicación le ofrece Santa Cruz?

3. Me gustaría saber más sobre sus relaciones con otras compañías. ¿Podría darme una descripción general de sus clientes? Además, me gustaría saber más sobre los productos y servicios que su empresa les presta.

4. Las empresas transnacionales, especialmente en el sector petrolero, son extremadamente poderosas. ¿En qué medida su trabajo está determinado por las preferencias de los clientes que acaba de mencionar?

5. ¿Qué actividades y proyectos quieren realizar en Santa Cruz en los próximos años? ¿Cuáles están previstos para otros lugares en Bolivia?

Guía: asociaciones empresariales/autoridades públicas

1. ¿Cómo describiría usted el rol de Santa Cruz en el sector petrolero, especialmente en cuanto a las empresas santacruceñas?

2. ¿Qué ventajas de ubicación ofrece Santa Cruz a las empresas involucradas en el sector petrolero?

3. ¿Qué organizaciones y programas han desarrollado usted y otros para apoyar al sector?

4. ¿En qué medida el sector petrolero ha contribuido a la industrialización de la economía boliviana/ santacruceña? ¿Cuáles son los obstáculos a este respecto?

Más allá de las entrevistas, el análisis se basa en artículos publicados en diarios bolivianos e informes de la Fundación Jubileo y la Fundación Milenio, dos organizaciones de la sociedad civil de Bolivia que publican sobre temas económicos, políticos y sociales. Se obtuvieron datos básicos sobre el sector petrolero de la Energy Information Administration estadounidense y por la página web A Barrel Full. Desafortunadamente, la mayoría de los conjuntos de datos y publicaciones disponibles se limita a asuntos como el volumen de exportaciones de petróleo y gas, la inversión en la exploración, así como las regalías y los impuestos que se derivan del sector. No hay publicaciones sustanciales sobre la industria de suministros. Dado que el análisis empírico, por lo tanto, se basa principalmente en entrevistas, tiene debilidades y fortalezas típicas de la investigación cualitativa: consigo explicar bien los mecanismos causales, mientras que admito que hay deficiencias en términos de cuantificación.

\section{Resultados}

En esta sección, se analizan primero las oportunidades para la industria boliviana de suministros, es decir, el rol de empresas santacruceñas como proveedores especializados. Después, se enfocan los tres procesos de desarticulaciones identificados por Murphy (2019), empezando con la degradación de dichas empresas por el modelo "llave en mano" y siguiendo con la expulsión y no participación de compañías locales de/en las redes de producción del sector petrolero.

\section{Oportunidades para los proveedores especializados}

En los años 1920, se encontró petróleo en Bolivia, cerca de la ciudad de Santa Cruz. Desde entonces, el sector -en términos de operadoras, así como proveedores bolivianos y extranjeros- se ha concentrado en esa ciudad (Scholvin, 2021), aunque los campos activos más grandes ahora estén a 600 kilómetros, en la provincia de Tarija (A Barrel Full, 2015; véase también: figura 1). Los hallazgos masivos de gas natural -que han reemplazado la soja como el bien más importante en las exportaciones bolivianas-se hicieron a fines del siglo pasado (Energy Information Administration, 2017). Están marcados por el mismo patrón geográfico.

Un entrevistado que trabaja para una operadora explicó que, debido a esta trayectoria histórica, hay empresas santacruceñas "con muchos años de experiencia". Cuentan con los conocimientos necesarios para llevar a cabo tareas especializadas, desde la colocación de gasoductos hasta la construcción de plantas de procesamiento de gas natural -en otras palabras, tareas que "no cualquiera puede hacer"-. Estas empresas tienen "personal altamente calificado" y "técnicos experimentados" (comunicación personal, 31 de julio, 2017). En cuanto a las corporaciones internacionales, no solo las leyes bolivianas les obligan a abrir sucursales en el país. Como se usa equipamiento muy pesado en la exploración y extracción de petróleo y gas, la presencia física es una necesidad práctica (comunicación personal, operadora, 8 de agosto, 2017).

El rol de Santa Cruz como nodo se ve reforzado por el hecho de que la ciudad sea el centro económico del país más allá del petróleo y gas (Fundación Milenio, 2018b), lo que significa que tiene una base industrial y 


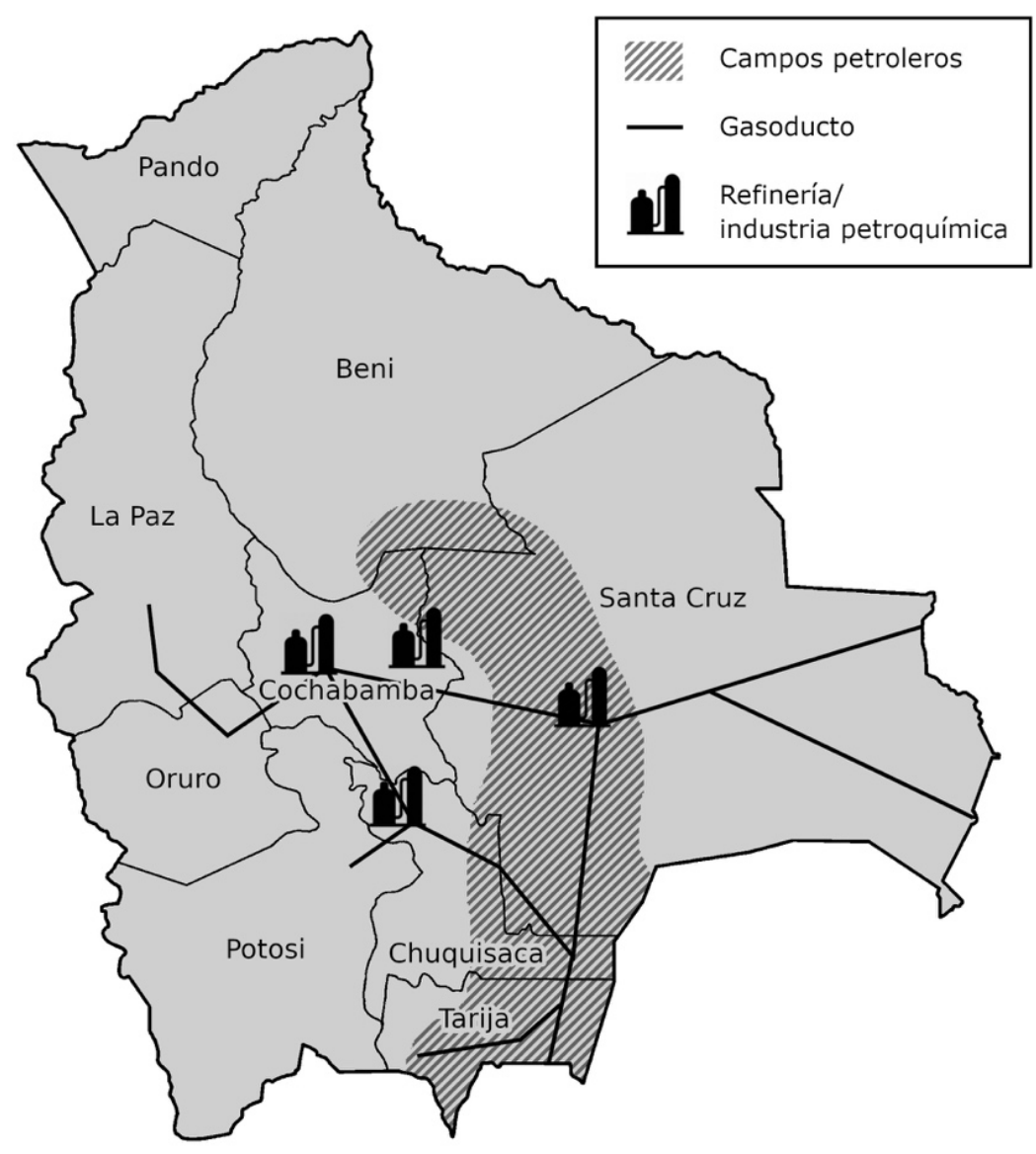

Figura 1. El sector petrolero en Bolivia. Fuente: Elaboración propia

una buena infraestructura de transporte que se conecta al nivel nacional e internacional (comunicación personal, petrolera nacional, 4 de agosto, 2017). Dos ejecutivos de una operadora subrayaron que, desde Santa Cruz, "es más sencillo trasladarse [...] a los campos [petroleros]". Por ese motivo y debido a los factores ya señalados, "es más natural tener un centro de negocios en Santa Cruz que en cualquier otra ciudad del país" (comunicación personal, 3 de agosto, 2017). El entrevistado mencionado en el párrafo anterior añadió que Santa Cruz "ofrece todas las ventajas para vivir bien, para trabajar bien" (comunicación personal, 31 de julio, 2017) - una fortaleza suave cuya importancia no se debe subestimar en un país en vías de desarrollo-. En otras palabras, las condiciones son favorables para que Santa Cruz y las empresas establecidas allá se vinculen a las redes globales y se beneficien considerablemente. Parece que puedan servir de proveedores especializados, lo que sugiere una forma del acoplamiento estratégico atractiva.
En efecto, un consultor explicó que varias compañías locales comenzaron como proveedores de materiales simples, requeridos por los proveedores especializados del extranjero. Esto les permitió adaptarse a los estándares del sector, adquiriendo nuevas habilidades $\mathrm{y}$, por lo tanto, incursionando en la construcción y el mantenimiento (comunicación personal, 7 de agosto, 2017). Directores y ejecutivos de empresas santacruceñas señalaron que después de haber trabajado en proyectos domésticos, sus actividades se expandieron hacia los países limítrofes, generalmente siendo contratados por las operadoras para quienes habían trabajado en Bolivia (comunicación personal, tres proveedores locales, 3 y 4 de agosto, 2017). La internacionalización no se limita a este modelo a babucha. Como una ejecutiva de una operadora subrayó, "el nombrar que hayan tenido un trabajo, una experiencia, con nosotros, también avala para [las compañías santacruceñas] puedan tener otro tipo de servicio con otra [operadora]" (comunicación personal, 8 de agosto, 2017), tanto en Bolivia, como en 
el extranjero. En cuanto al desarrollo regional, implica que debido a la vinculación a las redes globales, hay ingresos disponibles en Santa Cruz que provienen de operaciones en el extranjero y se pueden reinvertir localmente.

Entrevistados de una asociación empresarial confirmaron la relevancia de "tener clientes multinacionales en su currículum [es decir, el currículum de las empresas santacruceñas]". Agregaron que la contratación por corporaciones extranjeras ha causado un "cambio de cultura [laboral]" que ha resultado en el hecho de que las empresas y el personal de Bolivia sean altamente reconocidos en el país y afuera. Ellos relacionaron este desarrollo positivo a la privatización de YPFB en 1996 que "ayudó mucho a la industria de servicios [petroleros]. Primero porque la lógica de [las operadoras] es tercerizar. $\mathrm{Su}$ foco [es]: nosotros somos buenos para buscar hidrocarburos y explotar, pero todos los servicios alrededor, los tercerizamos. [...]. Adicional a eso, ayudó a crear un estándar de la industria, de exigencia, a nivel mundial" (comunicación personal, 1 de agosto, 2017).

\section{Degradación por el modelo llave en mano}

Sin embargo, la situación de las empresas santacruceñas es mucho más complicada que la sección anterior indica. Un hombre de negocios explicó que su compañía solía ser contratada para trabajos de construcción por las operadoras, así como por los proveedores de servicios especializados -Baker Hughes y Schlumberger, entre otros-. Usando la palabra inglesa, describió estas relaciones como "partnerships". Hoy, sus antiguos clientes contratan a epecistas, que generalmente son españolas o estadounidenses. Las epecistas luego subcontratan a proveedores santacruceños, como aquello del entrevistado, y los presionan para que reduzcan los costos (comunicación personal, 4 de agosto, 2017). Otro entrevistado contó que, en el pasado, su compañía proporcionaba módulos pequeños y medianos para proyectos más grandes, colaborando con otras empresas locales y siendo contratada directamente por las operadoras -"un modelo que funcionó [para todos]", dijo él-. Hoy, el 70 por ciento de su negocio es con epecistas, que el entrevistado llamó "un nuevo monstruo" (comunicación personal, proveedor local, 3 de agosto, 2017).

¿Por qué han surgido las epecistas? ¿Y por qué tienen una tan mala reputación? El término "epecista" viene de las palabras inglesas engineering, procurement, construction y management (en español, ingeniería, adquisiciones, construcción y gestión). Las epecistas -también denominadas "empresas EPCM"- ofrecen todos estos servicios a sus clientes. Creyendo trabajar más rentable de esta manera, las operadoras han decidido pasar a proyectos llave en mano en vez de contratar a numerosos proveedores locales para tareas puntuales. Significa que hay un solo contratista por proyecto y esta empresa debe proporcionar un paquete completo. Para las empresas locales, el problema con los proyectos llave en mano es que son tan complejos que su ejecución requiere seguros muy altos - para cubrir los costos potenciales de demoras o daños ambientales, por ejemplo- ${ }^{-}$. Las empresas bolivianas no tienen la capacidad financiera suficiente para pagar seguros de este tamaño (comunicación personal, asociación empresarial y proveedor local, 1 y 4 de agosto, 2017). Usando las palabras de un entrevistado, les "falta totalmente la capacidad financiera" (comunicación personal, proveedor local, 3 de agosto, 2017). Una ejecutiva de una operadora confirmó que "antes de invitarle formalmente, vemos si esta empresa [local] tiene una espalda financiera lo suficientemente gruesa"(comunicación personal, 8 de agosto, 2017). Como consecuencia, han surgido las epecistas.

Esta barrera de entrada se ve reforzada por la insuficiencia del sector bancario en Bolivia. Un consultor con experiencia previa en una epecista explicó que:

una garantía de una buena obra es el 7 por ciento [del precio total]. Entonces, si se construye una planta por [USD] 400 millones, 7 el por ciento son 28 millones. Ninguna empresa local puede tocar una boleta para una garantía por ese costo, principalmente por las condiciones que imponen los bancos. Los bancos te piden garantía de 1:1 [...]. Ese es el rol de las empresas extranjeras: vienen por la capacidad financiera. (comunicación personal, 31 de julio, 2017)

Esta reestructuración de las redes globales no significa, en la mayoría de los casos, que los proveedores santacruceños sean expulsados. Siguiendo el nuevo modelo,

estas empresas grandes [es decir, las epecistas] ponen la plata. Tienen el know-how [en ingeniería y construcción], por supuesto, pero lo que ellas hacen [es]: vienen a Bolivia y subcontratan a empresas locales. Por eso, al final, el trabajo [físico] lo hacen empresas bolivianas (comunicación personal, operadora, 31 de julio, 2017),

que son rebajadas de contratistas a subcontratistas y, como se señaló, sufren de una presión creciente para reducir los costos. Por lo demás, no hay aportes de las epecistas 
más allá del aspecto financiero y los contactos con las empresas líderes del sector. El consultor explicó que "el know-how, lo tenemos acá [...]. Todas las obras, las hacen ingenieros bolivianos y son las empresas extranjeras que envíen solamente supervisores" (comunicación personal, 31 de julio, 2017). Por lo tanto, las epecistas son "constructoras que no construyen [nada]" (comunicación personal, proveedor local, 4 de agosto, 2017), como lo expresó otro entrevistado.

Para proporcionar la seguridad financiera, los contactos con las empresas líderes y coordinar el trabajo de varios subcontratistas, las epecistas aparentemente agregan del 5 al 10 por ciento a los costos reales de cada proyecto, que luego se convierte en su ganancia (comunicación personal, consultor, 31 de julio, 2017). En otras palabras, la obra de USD 400 millones significa 20 a 40 millones para la epecista contratada. Mientras tanto, los proveedores locales asumen todos los riesgos de demoras, malas prácticas y asuntos similares (comunicación personal, dos proveedores locales, 3 de agosto, 2017). Las operadoras, por supuesto, exigen que las epecistas tengan seguros adecuados. Como se explicó, los seguros pesados son la razón del surgimiento del modelo epecista. Sin embargo, las epecistas imponen los mismos requisitos - cortados en piezas pequeñas que reflejan las tareas correspondientes-a los subcontratistas (comunicación personal, bróker, 8 de agosto, 2017). Si algo sale mal, las epecistas serán responsables frente a las operadoras, pero pasarán la responsabilidad directamente a las empresas santacruceñas.

\section{Expulsión de tareas complejas}

El modelo llave en mano, que explica la degradación del rol de las compañías santacruceñas, aparentemente también significa que estas empresas ahora se encuentran excluidas de algunas actividades. Ejecutivos de una operadora señalaron que, a las empresas locales, les faltan la maquinaria y la tecnología para proyectos a gran escala, y dijeron que "hay una ficción de que las empresas locales puedan substituir [los proveedores extranjeros]" (comunicación personal, 7 de agosto, 2017), lo que es verdadero, al menos bajo la condición de que las empresas líderes no estén dispuestas a volver a contratar a numerosos proveedores locales para tareas específicas. Otro entrevistado dijo con respecto a los proyectos llave en mano más grandes que "no hay compañías locales que hayan construido algo de este tamaño [...]. Normalmente, lo hacés con una [empresa] extranjera porque primero, experiencia y segundo, capacidad financiera" (comunicación personal, petrolera nacional, 4 de agosto, 2017).
Los representantes de la asociación empresarial mencionados más arriba también se mostraron escépticos en cuanto a las capacidades de las empresas santacruceñas o, más bien, a la sobreestimación de sus habilidades:

Las [empresas locales] dicen 'nosotros podemos absorber [este proyecto]', [pero] la capacidad económica de [estas empresas], por más que sea un grupo de empresas, con las garantías que pueden dar [...], te das cuenta de que no hay las condiciones y estamos hablando de proyectos de ni mucha tecnología, ni mucho know-how, pero de mucho riesgo. (comunicación personal, 1 de agosto, 2017)

Otro desafío es que las epecistas proporcionan todos los planes de construcción y el material que se necesita, expulsando así a los proveedores santacruceños que solían desempeñar los roles correspondientes. Por ese motivo y como consecuencia de la degradación (o el cálculo erróneo de los costos y riesgos de proyectos cada vez más grandes), muchas empresas santacruceñas se han declarado en bancarrota (comunicación personal, proveedor local, 3 de agosto, 2017). También parece haber corrupción o al menos relaciones dudosas que conducen a la exclusión de las empresas locales. Un entrevistado dijo que, desde la renacionalización, "el gobierno maneja todo [y] las epecistas se juntan, se pegan al gobierno" (comunicación personal, proveedor local, 3 de agosto, 2017). Es difícil encontrar pruebas que respalden tales afirmaciones. Sin embargo, YPFB se ha visto afectado por numerosos casos de corrupción y negocios ilícitos. Siete de los ocho presidentes de la estatal durante los catorce años de la presidencia de Evo Morales fueron acusados de corrupción. Uno de ellos, Santos Ramírez, fue condenado a doce años de prisión. Refiriéndose a la época de Morales, un consultor destacó que "hay mucha gente política adentro de YPFB, [gente] alogada por su afiliación política y no por su capacidad técnica [...], gente que nunca ha trabajado en el sector" (comunicación personal, 7 de agosto, 2017). Bajo el gobierno de la derecha que asumió después del golpe contra Morales a fines de 2019, YPFB ya ha tenido tres presidentes y uno de ellos se encontraba prófugo de la justicia cuando se entregó el manuscrito de este artículo.

Parece importante señalar que la expulsión es anterior al auge de las epecistas. Un bróker explicó que, con la privatización de YPFB, las compañías extranjeras establecieron los seguros como un principio para el sector. Según su experiencia, las empresas locales necesitan alrededor de USD 1 millón como garantía para un proyecto promedio con YPFB (es decir, una tarea de alcance limitado 
que debe coordinarse con el aporte de otros proveedores). Las operadoras extranjeras demandan seguros que cubren USD 2 millones. El entrevistado agregó que "son gastos que les duelen [a las empresas locales]" (comunicación personal, 8 de agosto, 2017) porque disminuyen la competitividad frente a los proveedores extranjeros. Por lo tanto, incluso si las operadoras estuvieran dispuestas a volver a contratar a numerosos proveedores santacruceños, muchos probablemente quedarían excluidos del sector.

\section{No participación por barreras de entrada y políticas contraproducentes}

Mientras que la expulsión es una forma de desarticulación que aplica a las empresas que alguna vez fueron parte de las redes globales, la no participación se refiere a todas aquellas que nunca lograron conectarse o, alternativamente, a los segmentos de las redes en consideración que nunca han tenido participación local. Además de las finanzas, los altos estándares del sector petrolero son una barrera de entrada a este respecto. Para las tareas más sofisticadas relacionadas con la exploración y extracción de hidrocarburos, no existen proveedores bolivianos (comunicación personal, operadora, 3 de agosto, 2017). No es sorprendente, considerando que los servicios correspondientes son prestados por menos de una docena de corporaciones globales: Baker Hughes, Halliburton, Schlumberger, Wintershall y pocas otras. Una entrevistada de una operadora agregó que varios insumos - tubos para gasoductos, por ejemplo- no están disponibles en el mercado boliviano (comunicación personal, 8 de agosto, 2017).

Incluso respecto a los servicios genéricos, existen importantes desafíos. Una entrevistada que trabaja para uno de los grandes proveedores especializados recién mencionados explicó que su empresa procura localmente servicios como limpieza de oficinas, seguridad y transporte. Encontrar proveedores suficientemente calificados no es fácil. La entrevistada agregó que la contratación en servicios de transporte-aviones pequeños, helicópteros, camiones y otros medios de transporte por carretera- es particularmente difícil porque no todos los proveedores están dispuestos a dedicar tiempo a programas de capacitación e invertir en vehículos que cumplen con requerimientos de la empresa de la entrevistada (comunicación personal, 8 de agosto, 2017). Otros brindaron el ejemplo de que los servicios de catering para los campamentos en ubicaciones dispares cerca de pozos de petróleo y gas deben cumplir con los mismos estándares que aplican al catering de aerolíneas internacionales (comunicación personal, operadora, 3 de agosto, 2017).
La transformación de las redes globales hacia el modelo llave en mano y el auge de las epecistas refuerzan estos problemas. Casi todos los entrevistados de las operadoras activas en Bolivia enfatizaron que están comprometidos con la mejora de las habilidades de sus proveedores, ofreciendo capacitaciones en contabilidad, protección del medio ambiente y estándares de calidad y seguridad (comunicación personal, 31 de julio, 3 y 8 de agosto, 2017). Sin embargo, las epecistas -que ahora tienen el contacto con los proveedores santacruceños en vez de las operadoras- operan bajo un modelo de negocio a corto plazo. Ejecutan proyectos según demanda de forma in-out, pero no invierten en Bolivia (comunicación personal, consultor, 31 de julio, 2017).

A este respecto, ciertamente no ayuda que el gobierno de Morales haya entendido el desarrollo basado en hidrocarburos exclusivamente como procesamiento doméstico. Un informe de la Fundación Jubileo (2017a) y un artículo de Ramírez Cendero (2014) resumen que YPFB y la Empresa Boliviana de Industrialización de Hidrocarburos (EBIH) -creada como compañía estatal en 2009- recibieron instrucciones de construir varios complejos petroquímicos. Además del lento progreso de estos proyectos, parece que no son competitivos al nivel internacional. Tampoco tienen una base de recursos suficiente por la falta de exploración (Fundación Jubileo, 2017b; Fundación Milenio, 2018a). Peor aún, EBIH e YPFB contrataron a epecistas para la ejecución de los proyectos, así reforzando la degradación, exclusión y no participación de los proveedores locales. El desarrollo de una industria de suministros, por su parte, ni siquiera se menciona en la estrategia de hidrocarburos del gobierno, aunque el documento tenga más de 500 páginas (Ministerio de Hidrocarburos y Energía, 2008).

\section{Conclusiones}

El presente artículo analizó las perspectivas del desarrollo regional a través del sector petrolero en Bolivia, concentrándose en la industria de suministros y aplicando unas herramientas clave del enfoque de las redes globales de producción. Para superar al sesgo inclusivo de este enfoque, se estudiaron tres procesos de desarticulaciones.

Debido a una larga trayectoria histórica, Santa Cruz es el centro del sector. Con las excepciones de EBIH e YPFB, no hay corporaciones bolivianas que sean líderes en las redes correspondientes, pero algunas empresas santacruceñas se benefician, empezando como proveedores de insumos genéricos y volviéndose proveedores especializados que, en varios casos, han logrado internacionalizar sus 
negocios. Este proceso ofrece ventajas considerables en términos de captura de valor y, por lo tanto, oportunidades con respecto al desarrollo regional. No obstante, las redes globales han cambiado últimamente, yendo en detrimento a las empresas santacruceñas. Las operadoras ya no contratan a varios proveedores locales para tareas puntuales. Prefieren soluciones llave en mano, es decir, proyectos completos realizados por un solo contratista. Como las empresas locales no pueden pagar los altos seguros para proyectos llave en mano, se encuentran subcontratadas -es decir, degradadas- por epecistas extranjeras que capturan mucho valor en desventaja de los subcontratistas. La degradación está ligada a la expulsión de las empresas locales de tareas complejas y lucrativas como, por ejemplo, la planificación ingeniera. Aparentemente, se ve reforzada por la corrupción que afecta, principalmente, YPFB.

Sería equivocado asociar todos los problemas de la industria de suministros con los asuntos recién mencionados. Como el sector petrolero se caracteriza por altas barreras de entrada, ya les es difícil a las empresas bolivianas de promover servicios tan genéricos como catering y transporte. Varios insumos industriales no están disponibles en el mercado nacional. La no participación resulta también de políticas contraproducentes que potencian los procesos de desarticulaciones, siguiendo al objetivo de industrializar el sector adentro del país en vez de apoyar la industria de suministros.

En resumen, el artículo mostró que hay desventajas y trampas en cuanto a la participación en las redes globales de producción. No obstante, existen también oportunidades considerables para las empresas santacruceñas. Por lo tanto, parece necesario revisar el concepto de desarticulaciones. Nos ofrece tanto una perspectiva crítica que -siguiendo los artículos de Bair \& Werner (2011), McGrath (2018) y Werner (2016) - dirige nuestra atención al lado oscuro de la geografía económica, como un marco heurístico desarrollado por Murphy (2019). Tendría que convertirse en una teoría para explicar bajo qué condiciones aplican los tres procesos de desarticulaciones.

\section{Agradecimientos}

La investigación de campo fue financiada por la Sociedad Alemana de Investigación (número del proyecto: 275355279).

\section{Referencias}

A Barrel Full. (2015). Bolivia oil and gas profile. http:// abarrelfull.wikidot.com/bolivia-oil-and-gas-profile

Andreucci, D. (2017). Resources, regulation and the state: struggles over gas extraction and passive revolution in Evo Morales's Bolivia. Political Geography, 61, 170180. https://doi.org/10.1016/j.polgeo.2017.09.003

Atienza, M., Aroca, P., Stimson, R. \& Stough, R. (2016). Are vertical linkages promoting the creation of a mining cluster in Chile? an analysis of the SMEs' practices along the supply chain. Environment and Planning C, 34(1), 171-187. https://doi. org/10.1177/0263774X15614708

Atienza, M., Lufin, M. \& Soto, J. (2021). Mining linkages in the Chilean copper supply network and regional economic development. Resources Policy, 70, 101154. https://doi.org/10.1016/j.resourpol.2018.02.013

Bair, J. \& Werner, M. (2011). Commodity chains and the uneven geographies of global capitalism: a disarticulations perspective. Environment and Planning A, 43(5), 988-997. https://doi.org/10.1068/a43505

Bair, J., Berndt, C., Boeckler, M. \& Werner, M. (2013). Dis/articulating producers, markets, and regions: new directions in critical studies of commodity chains. Environment and Planning A, 45(11), 2544-2552. https://doi.org/10.1068/a46297

Barratt, T. \& Ellem, B. (2019). Temporality and the evolution of GPNs: remaking BHP's Pilbara iron ore network. Regional Studies, 53(11), 1555-1564. https://doi.org/10.1080/00343404.2019.1590542

Carrizo, S. C. \& Ramousse, D. (2019). Dinámicas energéticas e integración regional en el noroeste Argentino y el sur Boliviano. Revista de Geografia Norte Grande, 45, 51-62. http://dx.doi.org/10.4067/ S0718-34022010000100004

Coe, N. M., Dicken, P. \& Hess, M. (2008). Global production networks: realizing the potential. Journal of Economic Geography, 8(3), 271-295. https://doi. org/10.1093/jeg/lbn002 
Coe, N. M. \& Yeung, H. W. (2015). Global Production Networks: Theorizing Economic Development in an Interconnected World. Oxford University Press.

Dicken, P., Kelly, P. F., Olds, K. \& Yeung, H. W. (2001). Chains and networks, territories and scales: towards a relational framework for analysing the global economy. Global Networks, 1(2), 89-112. https://doi. org/10.1111/1471-0374.00007

Energy Information Administration. (2017). Bolivia. https://www.eia.gov/international/analysis/country/ BOL

Fundación Jubileo. (2017a). Industrialización de los hidrocarburos. https://jubileobolivia.com/ Publicaciones/Documentos/industrializacion-de-loshidrocarburos

Fundación Jubileo. (2017b). ¿Qué pasó con la estrategia boliviana de hidrocarburos? https://jubileobolivia. com/publicaciones/Revistas-Especializadas/iQuepaso-con-la-estrategia-boliviana-de-hidrocarburos

Fundación Milenio. (2018a). El cuadro crítico de la producción y reservas de gas natural. https:// fundacion-milenio.org/coy-389-el-cuadro-critico-dela-produccion-y-reservas-de-gas-natural

Fundación Milenio. (2018b). Santa Cruz es la región que aporta al PIB del país con el 28,7\%. https:// fundacion-milenio.org/el-dia-santa-cruz-es-laregion-que-aporta-al-pib-del-pais-con-el-287

Gibbon, P. \& Ponte, S. (2005). Trading Down: Africa, Value Chains, and the Global Economy. Temple University Press.

Gobierno asegura que India plantea invertir 13.000 millones de dólares en gas. (2019a, marzo 29). Jornada. https://jornada.com.bo/gobierno-asegura-que-india-plantea-invertir-13-000-millones-dedolares-en-gas

Henderson, J., Dicken, P., Hess, M., Coe, N. M. \& Yeung, H. W. (2002). Global production networks and the analysis of economic development. Review of International Political Economy, 9(3), 436-464. https://doi.org/10.1080/09692290210150842
Hinojosa, L., Bebbington, A., Cortez, G., Chumacero, J. P., Humphreys Bebbington, D. \& Hennermann, K. (2015). Gas and development: rural territorial dynamics in Tarija, Bolivia. World Development, 73, 105-117. https://doi.org/10.1016/j.worlddev.2014.12.016

Hope, J. (2016). Losing ground? extractive-led development versus environmentalism in the Isiboro Secure Indigenous Territory and National Park (TIPNIS), Bolivia. The Extractive Industries and Society, 3(4), 922-929. https://doi.org/10.1016/j. exis.2016.10.005

Kaplinsky, R. (2000). Globalisation and unequalisation: what can be learned from value chain analysis? Journal of Development Studies, 37(2), 117-146. https://doi.org/10.1080/713600071

Kaplinsky, R. (2005). Globalization, Inequality and Poverty: Between a Rock and a Hard Place. Polity Press.

Kaplinsky, R. \& Morris, M. (2016). Thinning and thickening: productive sector policies in the era of global value chains. The European Journal of Development Research, 28(4), 625-645. https://doi. org/10.1057/ejdr.2015.29

Luna Acevedo, I. (2020, mayo 8). 11 cabezas de YPFB marcados por interinatos y corrupción. El Día. https:// www.eldia.com.bo/index.php?cat $=426 \&$ pla $=3 \& i d$ articulo $=304397$

Mackinnon, D. (2013). Strategic coupling and regional development in resource economies: the case of the Pilbara. Australian Geographer, 44(3), 305-321. https://doi.org/10.1080/00049182.2013.817039

Mackinnon, D., Dawley, S., Steen, M., Menzel, M. P., Karlsen, A., Sommer, P., Hansen, G. H. \& Normann, H. E. (2019). Path creation, global production networks and regional development: a comparative international analysis of the offshore wind sector. Progress in Planning, 130, 1-32. https://doi. org/10.1016/j.progress.2018.01.001

McGrath, S. (2018). Dis/articulations and the interrogation of development in GPN research. Progress in Human Geography, 42(4), 509-528. https://doi.org/10.1177/0309132517700981 
Milberg, W. \& Winkler, D. (2013). Outsourcing Economics: Global Value Chains in Capitalist Development. Cambridge University Press.

Ministerio de Hidrocarburos y Energía. (2008). Estrategia Boliviana de hidrocarburos. https:// www.hidrocarburos.gob.bo/phocadownload/ Estrategia $\% 20$ Boliviana $\% 20 \mathrm{de} \% 20$ Hidrocarburos\%202008.pdf

Morris, M., Kaplinsky, R. \& Kaplan, D. (2012). One Thing Leads to Another: Promoting Industrialisation by Making the Most of the Commodity Boom in Sub-Saharan Africa. Lulu.

Murphy, J. T. (2019). Global production network dis/ articulations in Zanzibar: practices and conjunctures of exclusionary development in the tourism industry. Journal of Economic Geography, 19(4), 943-971. https://doi.org/10.1093/jeg/lbz009

Ordenan aprehensión del expresidente de YPFB (2020, junio 19). Notibol. https://notibol.com/noticia/ bo/5eec669c28b73/ordenan-aprehension-delexpresidente-de-ypfb

Pellegrini, L. \& Ribera Arismendi, M. (2012). Consultation, compensation, and extraction in Bolivia after the 'left turn': the case of oil exploration in the north of La Paz department. Journal of Latin American Geography, 11(2), 103-120. https://doi. org/10.1353/lag.2012.0045

Perú y Bolivia acuerdan promover megapuerto en Ilo y uso compartido del gas. (2019b, junio 26). Jornada. https://jornada.com.bo/peru-y-bolivia-acuerdanpromover-megapuerto-en-ilo-y-uso-compartido-delgas

Phelps, N. A., Atienza, M. \& Arias, M. (2018). An invitation to the dark side of economic geography. Environment and Planning A, 50(1), 236-244. https:// doi.org/10.1177/0308518X17739007
Ramírez Cendero, J. M. (2014). Has Bolivia's 200612 gas policy been useful to combat the resource curse? Resources Policy, 41, 113-123. https://doi. org/10.1016/j.resourpol.2014.04.005

Scholvin, S. (2019). Vaca Muerta: perspectivas del desarrollo industrial en las redes globales de producción. Boletín Geográfico, 41(2), 81-96. http://revele.uncoma.edu.ar/htdoc/revele/index.php/ geografia/article/view/2541/59261

Scholvin, S. (2021). Ciudades en redes económicas: una comparación de Buenos Aires y Santa Cruz con Singapur, enfocada en el sector de petróleo y gas. Ciudad y Territorio [artículo por ser publicado].

Scholvin, S., Breul, M. \& Revilla Diez, J. (2020). A magic formula for economic development?: global market integration and spatial polarization in extractive industries. Area Development and Policy. https://doi.org/10.1080/23792949.2020.1823237

Van Grunsven, L. \& Hutchinson, F. E. (2016). The evolution of the electronics industry in Johor (Malaysia): strategic coupling, adaptiveness, adaptation, and the role of agency. Geoforum, 74, 7487. https://doi.org/10.1016/j.geoforum.2016.05.011

Werner, M. (2016). Global production networks and uneven development: exploring geographies of devaluation, disinvestment, and exclusion. Geography Compass, 10(11), 457-469. https://doi. org $/ 10.1111 /$ gec 3.12295

Yeung, H. W. (2009). Regional development and the competitive dynamics of global production networks: anEastAsianperspective. RegionalStudies, 43(3),325351. https://doi.org/10.1080/00343400902777059

Yeung, H. W. (2016). Strategic Coupling: East Asian Industrial Transformation in the New Global Economy. Cornell University Press. 